\title{
ISO observations of the interacting galaxy Markarian $297^{\star}$
}

\section{with the powerful supernova remnant 1982aa}

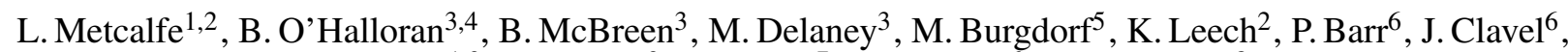 \\ D. Coia ${ }^{1,3}$, L. Hanlon ${ }^{3}$, P. Gallais ${ }^{7}$, R. Laureijs ${ }^{6}$, and N. Smith ${ }^{8}$
}

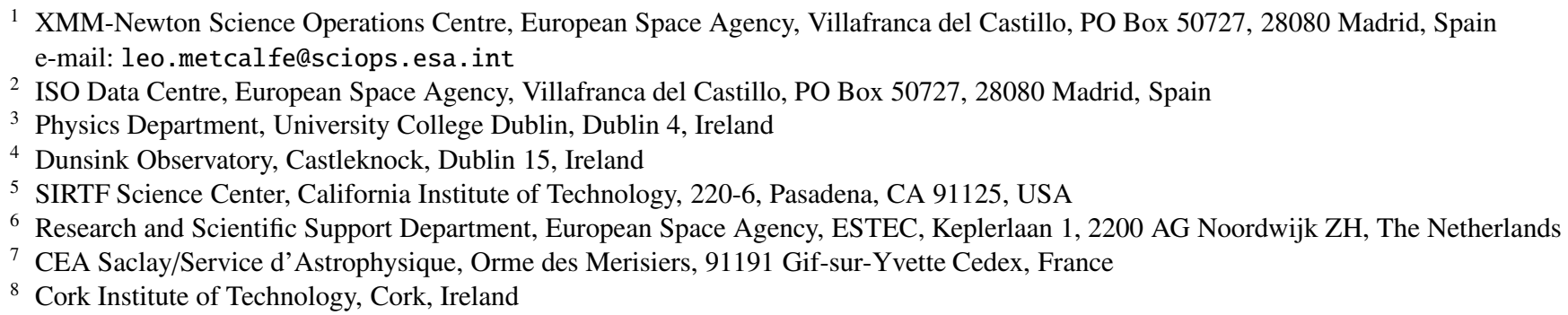

Received 17 October 2004 / Accepted 9 August 2005

\section{ABSTRACT}

Markarian (Mkn) 297 is a complex system comprised of two interacting galaxies that has been modelled with a variety of scenarios. Observations of this system were made with the Infrared Space Observatory (ISO) using the ISOCAM, ISOPHOT and LWS instruments. ISOCAM maps at $6.7 \mu \mathrm{m}, 7.7 \mu \mathrm{m}, 12 \mu \mathrm{m}$ and $14.3 \mu \mathrm{m}$ are presented which, together with PHT-S spectrometry of the central interacting region, probe the dust obscured star formation and the properties of the organic dust. The ISOCAM observations reveal that the strongest emission in the four bands is at a location completely unremarkable at visible and near-IR (e.g. 2MASS) wavelengths, and does not coincide with the nuclear region of either colliding galaxy. This striking characteristic has also been observed in the overlap region of the colliding galaxies in the Antennae (NGC 4038/4039), the intragroup region of Stephan's Quintet, and in IC 694 in the interacting system Arp 299, and again underlines the importance of infrared observations in understanding star formation in colliding/merging systems. At $15 \mu \mathrm{m}$, the hidden source in Mkn 297 is, respectively, 14.6 and 3.8 times more luminous than the hidden sources in the Antennae (NGC 4038/4039) and Stephan's Quintet. Numerical simulations of the Mkn 297 system indicate that a co-planar radial penetration between two disk galaxies yielded the observed wing formation in the system about $1.5 \times 10^{8}$ years after the collision. A complex emission pattern with knots and ridges of emission was detected with ISOCAM. The $7.7 \mu \mathrm{m}$ map predominantly shows the galaxy in emission from the $7.7 \mu \mathrm{m}$ feature attributed to PAHs (Polycyclic Aromatic Hydrocarbons). The $14.3 / 7.7 \mu \mathrm{m}$ ratio is greater than unity over most of the galaxy, implying widespread strong star formation. Strong emission features were detected in the ISOPHOT spectrum, while [O I], [O III] and [C II] emission lines were seen with LWS. Using data from the three instruments, luminosities and masses for two dust components were determined. The total infrared luminosity is approximately $10^{11} L_{\odot}$, which (marginally) classifies the system as a luminous infrared galaxy (LIRG). A supernova that exploded in 1979 (SN 1982aa) gave rise to one of the most powerful known radio remnants which falls close to the strongest mid-infrared source and is identified with star forming region 14 in the optical. This supernova explosion may have been accompanied by a gamma-ray burst (GRB), consistent with the idea that GRBs are associated with supernovae in star forming regions, and a search for a GRB consistent with the direction to Mkn 297, in satellite data from July to December 1979, is recommended.

Key words. galaxies: general - galaxies: interactions - galaxies: starburst - ISM: dust, extinction - infrared: galaxies

\section{Introduction}

Mkn 297 (also known as NGC 6052, Arp 209 and UGC 10182) has been the subject of many investigations because of its peculiar morphology (Weedman 1972). It has been referred to as a typical clumpy irregular galaxy (Taniguchi \& Tamura 1987) and a giant HII region galaxy (Thuan 1983; Thuan \& Martin 1981). It has a complex optical structure consisting of forty three identified bright optical knots within a common envelope
(Hecquet et al. 1987). The galaxy has no obvious companion and appears to be isolated. However, it has two distinct components revealed through optical spectroscopy (Duflot 1976; Burenkov 1988), CO (Sage et al. 1993) and HI line emission (Sofue et al. 1990), and consequently the system has been variously modelled as a colliding system consisting of two late-type spiral galaxies (Alloin \& Duflot 1979); the collision of a spiral galaxy with an irregular galaxy (Burenkov 1988); or more recently, using numerical $N$-body simulations, 
Table 1. The log of the ISO observations of Mkn 297. The nine columns list the TDT number (a unique identifier for an ISO observation), the AOT number (which identifies the observing mode used), the filter label, the wavelength range $(\Delta \lambda)$, the reference wavelength of the filter where appropriate, the duration of the measurement (including both the on- and off-source measurements), the field-of-view of the instrument in the configuration employed, the positional offset of the background measurement(s) with respect to the target reference position, and any additional notes.

\begin{tabular}{|c|c|c|c|c|c|c|c|c|}
\hline $\mathrm{TDT}^{a}$ & AOT\# & Filter & $\begin{array}{c}\Delta \lambda \\
(\mu \mathrm{m})\end{array}$ & $\begin{array}{c}\lambda_{\mathrm{ref}} \\
(\mu \mathrm{m})\end{array}$ & $\begin{array}{l}\Delta \mathrm{t} \\
(\mathrm{s})\end{array}$ & $\begin{array}{c}\text { FOV } \\
\prime \prime\end{array}$ & $\begin{array}{c}\text { Chop } \\
\text { " }\end{array}$ & Notes \\
\hline 47400671 & CAM03 & LW2 & $5.0-8.5$ & 6.7 & 588 & $48\left(1.5^{\prime \prime} /\right.$ pixel $)$ & 90 & chop in Dec \\
\hline 47400671 & CAM03 & LW6 & $7.0-8.5$ & 7.7 & 588 & 48 & 90 & chop in Dec \\
\hline 47400671 & CAM03 & LW3 & $12.0-18.0$ & 14.3 & 588 & 48 & 90 & chop in Dec \\
\hline 47400671 & CAM03 & LW10 & $8.0-15.0$ & 12.0 & 588 & 48 & 90 & chop in Dec \\
\hline 09101068 & CAM01 & LW3 & $12.0-18.0$ & 14.3 & 3794 & $480\left(6^{\prime \prime} /\right.$ pixel $)$ & & raster $^{b}$ \\
\hline 47400567 & PHT40 & SS/SL & $2.5-11.6$ & & 1024 & 25 & $+/-150$ & triang. chop ${ }^{c}$ \\
\hline 62702069 & LWS01 & & $43.0-190.0$ & & 1124 & 101 & & on-source \\
\hline 62702070 & LWS01 & & $43.0-190.0$ & & 1124 & 101 & see below ${ }^{a}$ & off-source \\
\hline
\end{tabular}

${ }^{a}$ All observations refer to target position R.A. $16^{\mathrm{h}} 05^{\mathrm{m}} 13.2^{\mathrm{s}}$ and Dec $+20^{\circ} 32^{\prime} 32.0^{\prime \prime}$ except for the LWS background measurement made at $16^{\mathrm{h}} 05^{\mathrm{m}} 57.2^{\mathrm{s}}+20^{\circ} 26^{\prime} 23.7^{\prime \prime}$.

${ }^{b}$ TDT 09101068 was a $4 \times 4$ raster with $96^{\prime \prime}$ step size.

${ }^{c}$ The term "chop" has been used to refer to all off-target measurements, although only PHT employed a chopping device. For the other instruments the spacecraft pointing was offset.

as the collision of two disk galaxies (Taniguchi \& Noguchi 1991). Throughout this paper we will refer to the component identified as a spiral by Burenkov (1988) as galaxy A and the other component as galaxy B.

The two components have a velocity separation of about $200 \mathrm{~km} \mathrm{~s}^{-1}$ and modelling reveals that their closest approach occurred about $1.5 \times 10^{8}$ years ago (Taniguchi \& Noguchi 1991). Mkn 297 has similar infrared properties to the Antennae Galaxies (NGC 4038/4039) and, as in the Antennae, the strongest infrared source does not coincide with the nuclei of the interacting galaxies (Vigroux et al. 1996; Kunze et al. 1996; Mirabel et al. 1998) nor, indeed, with any other feature notable at visual or near-IR wavelengths (see Figs. 1-3 below). Heeschen et al. (1983) have found a peculiar compact and variable radio source, Mkn 297A, lying several arcseconds north of the nuclei of the interacting galaxies (see Fig. 8). They interpreted the compact source as a complex of supernova remnants associated with a region of vigorous star formation. Yin \& Heeschen (1991) and Deeg et al. (1993) interpret Mkn 297A as a single luminous radio supernova which exploded in July 1979. Mkn 297 was observed by ROSAT, and has an X-ray luminosity $L_{X}=2 \times 10^{41} \mathrm{erg} \mathrm{s}^{-1}$ (Hensler et al. 1997) with ROSAT PSPC around $1 \mathrm{keV}$, a value that is comparable to the cumulative flux of the Antennae observed with Chandra at similar energy (Zezas et al. 2002).

The capabilities of the Infrared Space Observatory (ISO) (Kessler et al. 1996) permitted observations of Mkn 297 across a wide range of IR wavelengths with three complementary instruments. The observations and data reduction techniques are presented in Sect. 2. The results are presented in Sect. 3 and discussed in Sect. 4. A value of $H_{0}=72 \mathrm{~km} \mathrm{~s}^{-1} \mathrm{Mpc}^{-1}$ has been adopted and yields a distance of $66 \mathrm{Mpc}$ to Mkn 297.

\section{Observations and data reduction}

The ISO observations were obtained using: (a) the mid-infrared camera ISOCAM (Cesarsky et al. 1996), mainly in beamswitching mode, though one larger-area raster observation has been used; (b) the spectrometric channel (PHT-S) of the ISO photopolarimeter (ISOPHOT) (Lemke et al. 1996) in triangular chopping mode and (c) the medium-resolution grating mode of the long wavelength spectrometer LWS (Clegg et al. 1996) employing dedicated on- and off-source measurements. The parameters of these observations are listed in Table 1 and discussed in the following sections.

\subsection{ISOCAM}

A detailed technical description of the ISOCAM instrument and its observing modes can be found in (Blommaert et al. 2003). For the present work the $1.5^{\prime \prime}$ per pixel plate scale of the instrument has been used yielding a field of view of $48^{\prime \prime} \times 48^{\prime \prime}$. As explained in Sect. 2.4 an available lower-resolution 6"/pixel map has been used only to rule out the presence of very extended mid-IR emission from the system. Observations were obtained in four filters, with reference wavelengths (Moneti et al. 1997) at $6.7 \mu \mathrm{m}$ (LW2), $7.7 \mu \mathrm{m}$ (LW6), $12 \mu \mathrm{m}$ (LW10) and $14.3 \mu \mathrm{m}$ (LW3). Each filter observation consisted of two measurements, one centred $1.5^{\prime}$ away from the target, and the other centred on the target coordinates. Each of these beams was observed for 140 on-chip integrations of $2.1 \mathrm{~s}$ each, for a total dwell-time per filter of $588 \mathrm{~s}$. The diameter of the ISO point spread function (PSF) central maximum, in arcseconds, at the first Airy minimum is $0.84 \times \lambda(\mu \mathrm{m})$. The $F W H M$ is about half that, and Okumura (1998) obtained a value of $2.3^{\prime \prime}$ at $6.7 \mu \mathrm{m}$ 
and $4.6^{\prime \prime}$ at $14.3 \mu \mathrm{m}$ for the PSF $F W H M$ in the $1.5^{\prime \prime}$ per pixel configuration.

All data processing used the CAM Interactive Analysis (CIA) software (Ott et al. 1997, 2001; Delaney et al. 2002), as follows: (i) dark subtraction was performed using a dark model with correction for slow drift of the dark-current throughout the mission; (ii) glitch effects due to cosmic rays were removed following the method described in Starck et al. (1998a); (iii) transient correction for signal attenuation due to the lag in the detector response was performed by the method described by Abergel et al. (2000); (iv) the maps were flat-fielded using library flat-fields; (v) pixels affected by glitch residuals and other persistent effects were manually suppressed; (vi) the on-target and off-target beams were subtracted and (vii) the images were deconvolved using a multi-resolution transform method (Starck et al. 1998b).

Considerable care was needed in the preparation of the $14.3 / 6.7 \mu \mathrm{m}$ ratio map. Apart from deconvolution and background subtraction (O'Halloran et al. 2002), it was necessary to eliminate any misalignment between the 14.3 and $6.7 \mu \mathrm{m}$ images due mainly to some jitter ${ }^{1}$ in the positioning of the CAM wheels, leading to small offsets in astrometry. This was done by choosing a suitable reference source in both images and locating its position using a Gaussian fit. The coordinates of the reference object in each image were established at sub-pixel accuracy by the fit and the vector distance between the reference source positions in successive images was determined, establishing the extent of misalignment. A PSF was chosen, from a library of model PSFs, offset from the centre by the negative of the distance vector. This PSF was used to deconvolve and align the misaligned images. The reference image was then deconvolved with a centred PSF so the resultant images were aligned and had the same beam. Noise spikes in the ratio map, due to the division of background pixels having noise-related low apparent signal values were suppressed by applying the same positive threshold to the background pixels in both images before division. Note that photometry of sources was performed on the un-deconvolved maps using the CIA routine XPHOT, employing aperture photometry scaled for the PSF.

The durations of the observations in Table 1 include instrumental, but not spacecraft, overheads. The flux densities quoted in Table 2 for four source regions assume a spectrum having $\lambda F_{\lambda}=$ const., and have a photometric accuracy of about $\pm 15 \%$.

\subsection{PHT-S}

PHT-S consists of a dual grating spectrometer with a resolving power of 90 (Laureijs et al. 2003). Band SS covers the range $2.5-4.9 \mu \mathrm{m}$, while band SL covers the range 5.8-11.6 $\mu \mathrm{m}$. The PHT-S spectrum of the core region of the Mkn 297 system was obtained by operating the $24^{\prime \prime} \times 24^{\prime \prime}$ aperture of PHT-S in triangular chopping mode using the ISOPHOT focal-plane chopper. In this mode, the satellite pointed to the given target coordinates centred between two off-source-positions, and the chopper moved alternately from the target to the first off-source

\footnotetext{
1 Some "play" was designed into the CAM cryo-mechanisms to avoid mechanism seizure at LHe temperatures.
}

Table 2. The ISOCAM flux densities for MIR clumps in Mkn 297. The photometry was performed before deconvolution and is accurate to about $\pm 15 \%$.

\begin{tabular}{rccccc}
\hline \hline$\lambda$ & $\begin{array}{c}\text { Total flux } \\
\text { density } \\
(\mu \mathrm{m})\end{array}$ & \multicolumn{5}{c}{ Source flux densities } \\
& & 1 & 2 & 3 & 4 \\
\hline 6.7 & 141 & 19.5 & 16.0 & 14.0 & 19.0 \\
7.7 & 246 & 30.5 & 19.5 & 19.0 & 29.5 \\
12.0 & 269 & 42.5 & 28.0 & 24.5 & 31.5 \\
14.3 & 289 & 66.5 & 48.5 & 30.5 & 43.5 \\
\hline
\end{tabular}

position, back to the target and then to the second off-source position. Consequently, $50 \%$ of the exposure time is on target and $50 \%$ is distributed equally over the two off-source positions. In the on-target phase of the chop the PHT-S aperture was centred on the centre of the ISOCAM pointing, a position about 3 arcsec East of the centre of the $15 \mu \mathrm{m}$ image presented in Fig. 1, as indicated in the figure. The $24^{\prime \prime} \times 24^{\prime \prime}$ PHT-S aperture covered completely ISOCAM source regions 1, 2 and 4, and vignetted slightly the other regions. (See Sects. 2.4 and 3.3 below.)

PHT data processing was performed using the ISOPHOT Interactive Analysis (PIA) system, version 8.1 (Gabriel 2002). Data reduction consisted primarily of the removal of instrumental effects such as ionising particle impacts which result in a spurious increase in two or more consecutive read-out voltage values. The disturbance is usually very short and the slope of the integration ramp after a glitch is similar to the slope before it. Further reduction of the spectrum was performed by manual deglitching, where the slopes of the ramps were considered for a given PHT-S channel, and two possible corrections were applicable. First a strong startup transient was searched for (Acosta-Pulido et al. 2000) and, if present, suppressed by deleting either the initial 64 or $128 \mathrm{~s}$ of integration. The remaining signals were inspected for large deviations from the mean, indicating strong cosmic ray hits which could not be removed by the automatic deglitching. These anomalous signals were suppressed manually by masking data affected by the glitch. The mean differential signal (i.e. the difference of the on-source and off-source chopper beams) for each of the 128 pixels of PHT-S was derived. To absolutely calibrate the resulting spectrum it was scaled to match the signal level of the ISOPHOT-S Highly Processed Data Products for this target which can be found in the ISO archive (Richards \& Klaas 2002) associated with the Mkn 297 archive entry for this observation. The HPDP are somewhat noisier than our PIA produced product, but benefit from being part of a systematic reprocessing of a large sample of PHT-S spectra, and we consider that they are likely to have a better overall calibration. Relative (from point to point in the spectrum) and absolute (overall normalisation of the spectrum to flux standards) spectrometric accuracies of better than $10 \%$ are indicated. The results were plotted to obtain the spectrum for the region of Mkn 297 in the PHT-S aperture. Flux values derived from this spectrum have to be further 


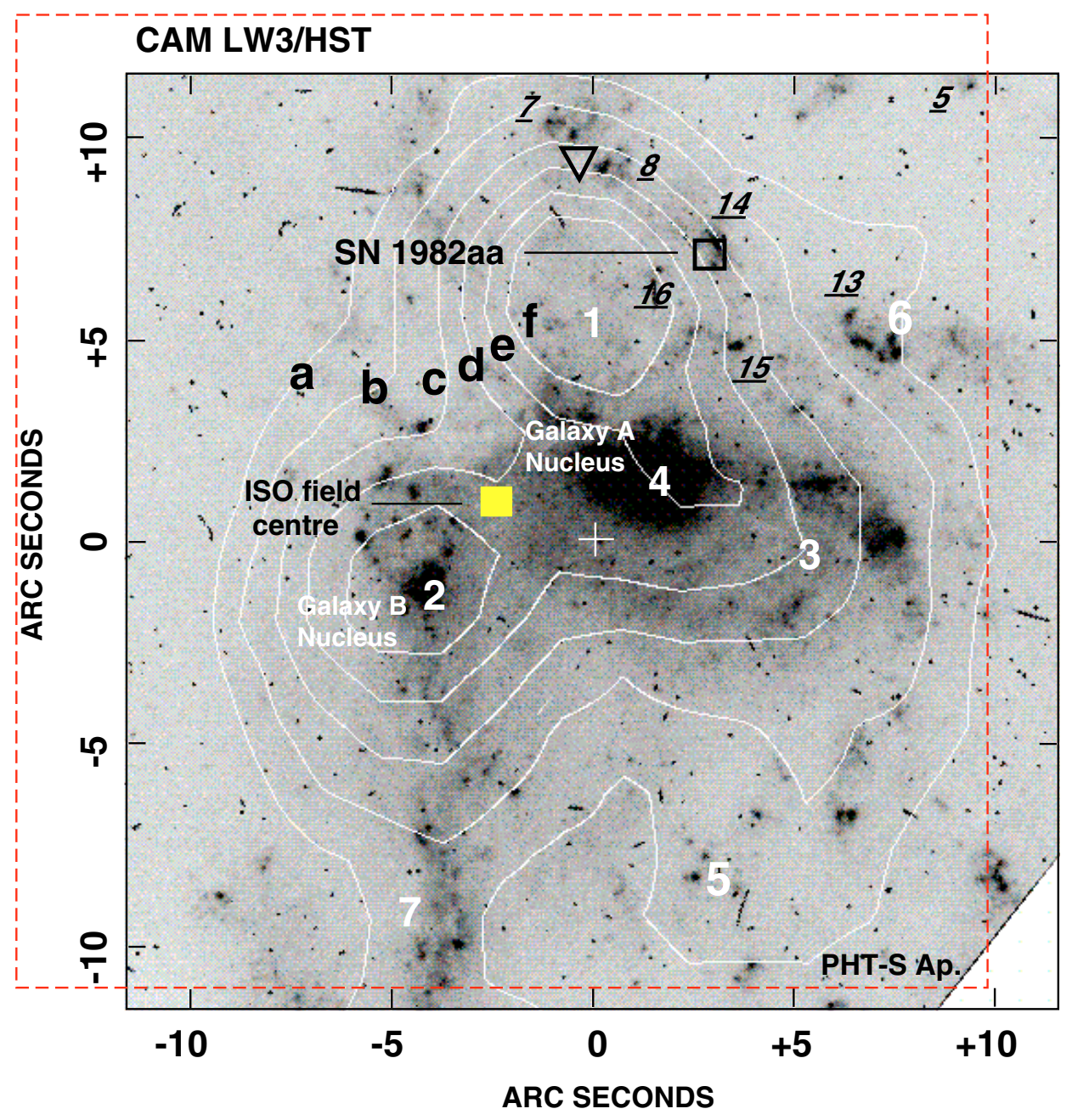

\section{MAP CENTRE + : R.A. 160513.0 DEC +20 3230.5}

Fig. 1. A deconvolved ISOCAM $14.3 \mu \mathrm{m}$ contour map of Mkn 297 overlaid on a HST image (Filter: $F 555 W$, Centre: $5407 \AA$, Bandwidth: $1236 \AA$ ). The contour levels, in the order a to f, are as follows (in units of $\mathrm{mJy} / \operatorname{arcsec}^{2}$ ): $0.7,1.0,1.5,2.0,2.5$ and 3.0. The seven regions identified by white numbers correspond to the positions of well-defined structures or extensions of emission seen in one or more of the bandpasses presented in Fig. 2. Small black underlined numbers identify some $\mathrm{H}_{\alpha}$ emission knots as designated by Hecquet et al. (1987), and help relate this map to earlier published data. Source 4 coincides approximately with the nuclear region of galaxy A and source 2 with the nuclear region of galaxy B, oriented north-south. However the strongest source, No. 1, does not correspond to either nucleus. The open square denotes the position of one of the brightest known radio supernovae, SN 1982aa (Mkn 297A) and corresponds to $\mathrm{H}_{\alpha}$ source 14. The position of a knot of 2MASS $K$-band emission is indicated by the small black triangle (see also Fig. 3 ). The filled light-coloured square designates the position of the centre of the PHT-S aperture, and the large dashed square on which it is centred indicates the size of the PHT-S aperture. The LWS aperture had the same centre, but was much larger than the region shown here.

scaled, as described in Sect. 2.4 below, to yield values more appropriate to the galaxy as a whole.

\subsection{Long Wavelength Spectrometer (LWS)}

A detailed technical description of the LWS instrument and its observing modes can be found in (Gry et al. 2003). A LWS spectrum of Mkn 297 spanning the range from 45 to $180 \mu \mathrm{m}$ was obtained with the LWS aperture centred on the ISOCAM map, at a position about $3^{\prime \prime}$ East of the nucleus of galaxy A, as indicated by the filled square in Fig. 1. The beam of LWS was slightly elliptical and its FWHM varied between $65^{\prime \prime}$ and $85^{\prime \prime}$, depending on wavelength and direction
(Swinyard et al. 1998). It was assumed that the source was completely included in the beam of the LWS instrument, so that no extended-source correction was necessary.

Because of an expected significant background signal in the LWS aperture due to combined Zodiacal and Cirrus emission in this sky region, a dedicated background measurement was made with the LWS aperture centred about 12 arcmin away from the on-source measurement (at a position determined to be of similar background based on the IRAS maps), and the resulting background was subtracted from the on-target case.

The grating was scanned 6 times over the entire wavelength range. The spectral sampling was set to give 4 samples per resolution element in each of the scans. 
We made use of the LWS Highly Processed Data Products (HPDP) for this target, available from the ISO archive (Lloyd et al. 2003). Because of the small flux from the source and the resulting poor signal-to-noise ratio of the LWS continuum spectrum, the data were rebinned to one point for each of the 10 LWS detectors, employing a scan-averaging method described by Sidher et al. (2000), yielding 10 samples of the continuum spanning the LWS spectral range and plotted in Fig. 7.

\subsection{Handling of different instrument fields-of-view}

The rather different apertures, or fields-of-view, of the instruments warrant comment. Observatory pipeline products from the low-resolution ISOCAM 6"/pixel raster observation of ISO TDT 09101068 have been used to establish that substantially less than $10 \%$ of the total galaxy MIR light falls outside of the $1.5^{\prime \prime} /$ pixel, $48^{\prime \prime}$ field-of-view upon which the results reported here rely. The much larger LWS aperture (see Sect. 2.3 above) is assumed to have covered the full extent of the galaxy, even at the longer wavelengths addressed by LWS. The PHT-S aperture is a square $24^{\prime \prime}$ on a side, and was placed as shown in Fig. 1. It certainly missed some of the integrated galaxy signal in the PHT-S wavelength range, although it included most of the knots of mid-infrared emission found in the ISOCAM map. But the PHT-S aperture has a vignetted profile (Laureijs et al. 2003). So some scaling has to be applied to PHT-S signals to adapt them to the signals that would have been recorded in corresponding spectral ranges over the full area of the ISOCAM footprint. To determine the scaling factor the PHT-S spectrum was used to synthesise a $7.7 \mu \mathrm{m}$ (LW6) ISOCAM bandpass measurement by integrating the CAM filter profile over the PHT-S spectrum. The result was compared with the CAM 7.7 micron global galaxy photometry and found to differ by a factor of 1.46. PAH feature strengths derived from the PHT-S spectrum were scaled by this factor (Sect. 3.3).

\section{Results}

\subsection{A hidden source}

The deconvolved $14.3 \mu \mathrm{m}$ map of Mkn 297 overlaid on a HST image (filter $F 555 W$ at $5407 \AA$ ) is presented in Fig. 1, while the deconvolved maps at $6.7,7.7,12$ and $14.3 \mu \mathrm{m}$ are presented in Figs. 2i-2iv. The figures reveal a complex emission pattern of overlapping sources and seven source regions are numbered on the maps. The integrated CAM flux densities for the system are presented in Table 2 along with estimated flux densities from the four brightest MIR clumps. Knots that appear well defined in one bandpass may not be so sharp in another (see Fig. 2). Representative flux-density values have been derived by performing aperture photometry about the locations numbered in 1 to 4 in Figs. 1 and 2 and scaling each result to the PSF that would deliver the same signal in the aperture used. Where no well-defined point-source appears in some bandpass these "point-source" flux densities nevertheless serve to characterise relative brightness over the map. Source 4 coincides approximately with the nuclear region of galaxy A and source 2 with the nuclear region of galaxy B. The strongest source, labeled 1 on all the CAM maps, does not coincide with the nucleus of either galaxy but is displaced by about $2 \mathrm{kpc}$ from the nucleus of galaxy A and peaks over a region devoid of optical emission knots. (We stress, by the way, that this source is clearly distinguished also in the un-deconvolved maps.) Hecquet et al. (1987) reported numerous optical emission knots in Mkn 297, and knots 7 and 8 in their numbering scheme have the second and third highest values of $B-R$ (1.8 and 2.3 respectively) for the system and are within a few arcseconds of the mid-infrared peak. Knot 14 is the site of the variable radio source reported by Heeschen et al. (1983). These optical knots may represent the visible portions of a much vaster obscured starburst. A similar result has been obtained (Vigroux et al. 1996; Mirabel et al. 1998) for the interacting galaxies NGC 4038/4039 (The Antennae) where the strongest mid-infrared source does not coincide with either of the nuclei of the constituent galaxies.

In order to compare the ISOCAM map with previously published results at other wavelengths (Heeschen et al. 1983; Hecquet et al. 1987; Lonsdale et al. 1992; Deeg et al. 1993, 1997) it is necessary to consider some inconsistencies in the published astrometry of features in the Mkn 297 system. The first three of the above-referenced papers use astrometry reported by Heeschen et al. (1983), derived ultimately from glass copies of original photographic plates and attributed in Hecquet et al. (1987) to a private communication from Casini in 1980. However, a careful comparison of the Hecquet et al. (1987) astrometry (their Table 1 and Fig. 2b) with 2MASS and HST images reveals a roughly 6 " offset of their coordinates to the North West. The resulting positions for optical knots in Mkn 297 differ systematically from positions derived by Deeg et al. (1993) based on independent CCD photometry. Similarly, the radio supernova reported by Heeschen et al. (1983) and Yin (1994), which from Figs. 1, 2 and 8 of this paper clearly corresponds to $\mathrm{H}_{\alpha}$ source 14 of Hecquet et al. (1987), only falls at that location subject to the above adjustment of the astrometry of Hecquet et al. (1987). The astrometry of Casini, Heeschen et al. (1983), Yin (1994) and Deeg et al. (1993) appears to be mutually consistent with all recent imaging data. In fact, Lonsdale et al. (1992) noted the same discrepancy and refer to a private communication from Hecquet \& Coupinot(1991) in which they concur.

Figure 1 therefore adopts the 2MASS astrometry referenced to ISOCAM source 4, assumed to correspond to the brightest knot in the 2MASS $K$-band data. This places ISO source 2 over the nucleus of galaxy B. Figure 3 presents an overlay of the ISO $15 \mu \mathrm{m}$ (white contours) and 2MASS $K$-band (black contours) on the HST image of Mkn 297 used in Fig. 1. It is clearly seen that ISO source 1 is not evident in the 2MASS map. (See also the $J, H$ and $K$ maps published by Smith et al. (1996).) The $K$-band data of Cairós et al. (2003) also show a source roughly 4 arcsec North of our source 1 and consistent with the location of the 2MASS source and the optical knots.

Within the accuracy of the various sources of positional information used to construct Fig. 1 these several detections are consistent with a single extended emitting region suffering, at different positions, different degrees of extinction. 

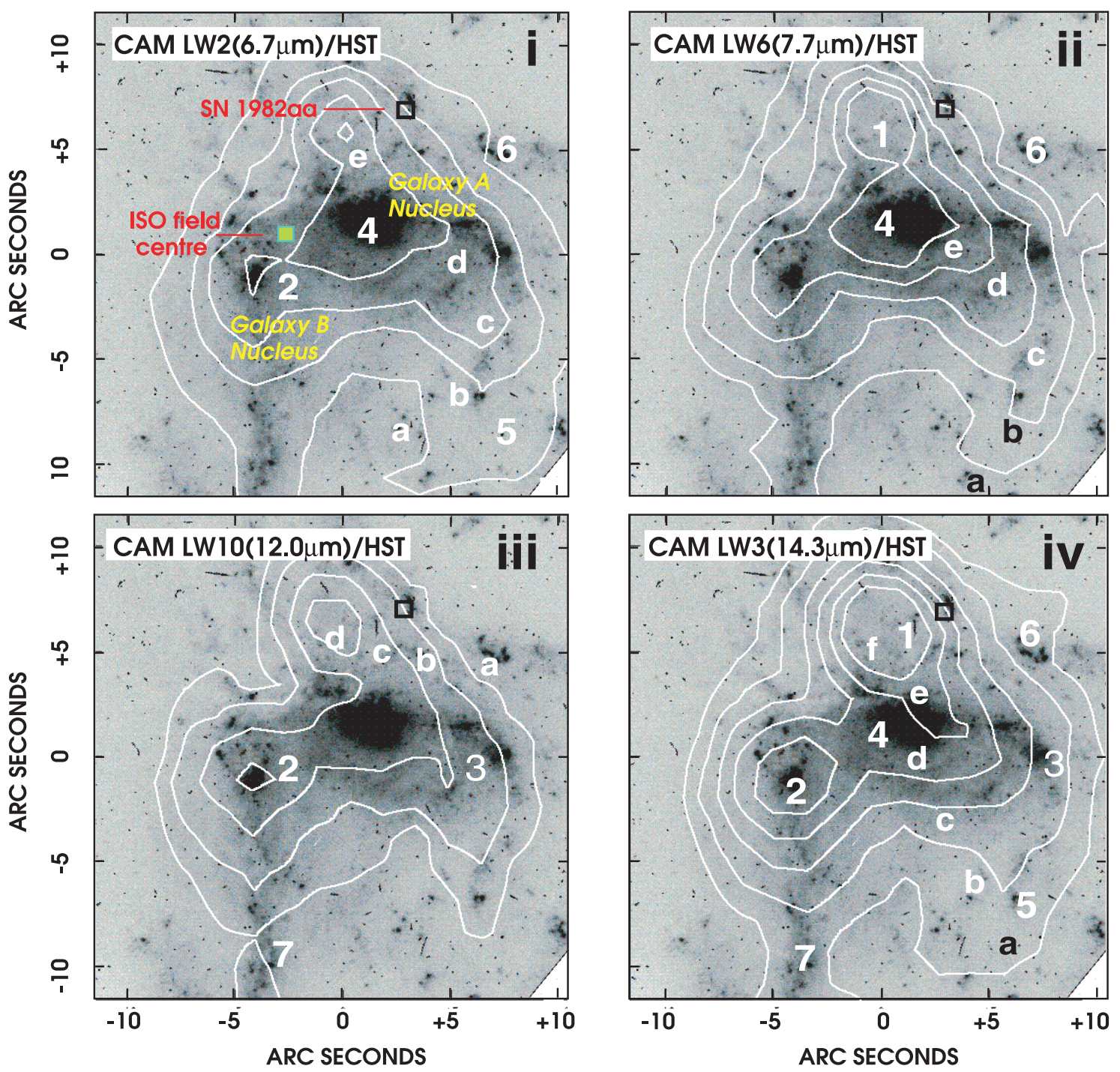

Fig. 2. Deconvolved ISOCAM contour maps overlaid on the HST image: i) $6.7 \mu \mathrm{m}$, ii) $7.7 \mu \mathrm{m}$, iii) $12 \mu \mathrm{m}$ and iv) $14.3 \mu \mathrm{m}$ maps are presented. Seven regions of emission are identified (the numbers running from 1 to 7). Sources 2 and 4 coincide with the nuclear regions of the constituent interacting galaxies. The contour levels in the sequence a to $\mathrm{f}$ are as follows (in units of $\mathrm{mJy} / \operatorname{arcsec}^{2}$ ): i) $0.4,0.7,1.0,1.5$ and 2.0 ; ii) $0.7,1.0$, $1.5,2.0$ and 2.5 ; iii) $0.7,1.0,1.5$ and 2.0 ; iv) $0.7,1.0,1.5,2.0,2.5$ and 3.0 .

\subsection{Four ISOCAM bandpasses}

Figure 2 presents the $6.7 \mu \mathrm{m}, 7.7 \mu \mathrm{m}, 12 \mu \mathrm{m}$ and $14.3 \mu \mathrm{m}$ ISOCAM maps. The $7.7 \mu \mathrm{m}$ map essentially shows the galaxy in the emission of a spectral feature usually attributed to polycyclic aromatic hydrocarbon (PAH) emission. The $14.3 \mu \mathrm{m}$ map includes contributions from warm dust, nebular line emission such as [NeII] and PAH feature emission. The galaxy has the approximate shape of an inverted tuning fork with regions $1,2,3$ and 4 dominating the emission to different degrees at different wavelengths. IRAS flux densities for the system are given in Table 3 for reference.

The 14.3/6.7 $\mu$ m ratio map presented in Fig. 4 shows values greater than unity across much of the map, which is indicative of active star formation over a wide area. The $14.3 / 6.7 \mu \mathrm{m}$ ratio generally decreases as interactions develop and starbursts age
Table 3. IRAS flux densities for Mkn 297 (from Sanders et al. 2003). The four columns list the filter used, the wavelength range, the reference wavelength for the filter and the flux density measured.

\begin{tabular}{lccc}
\hline \hline Filter & $\begin{array}{c}\Delta \lambda \\
(\mu \mathrm{m})\end{array}$ & $\begin{array}{c}\lambda_{\text {ref }} \\
(\mu \mathrm{m})\end{array}$ & $\begin{array}{c}\text { Total flux density } \\
(\mathrm{mJy})\end{array}$ \\
\hline IRAS $12 \mu \mathrm{m}$ & $8.5-15$ & 12 & $280 \pm 22$ \\
IRAS $25 \mu \mathrm{m}$ & $19-30$ & 25 & $830 \pm 27$ \\
IRAS $60 \mu \mathrm{m}$ & $40-80$ & 60 & $6790 \pm 45$ \\
IRAS $100 \mu \mathrm{m}$ & $83-120$ & 100 & $10570 \pm 340$ \\
\hline
\end{tabular}

because PAHs, which dominate the shorter-wavelength band, are no longer destroyed by the highly ionizing O-stars, and dust emission declines (Vigroux et al. 1999; Cesarsky \& Sauvage 1999; Charmandaris et al. 1999; Helou 1999). The ratio is 


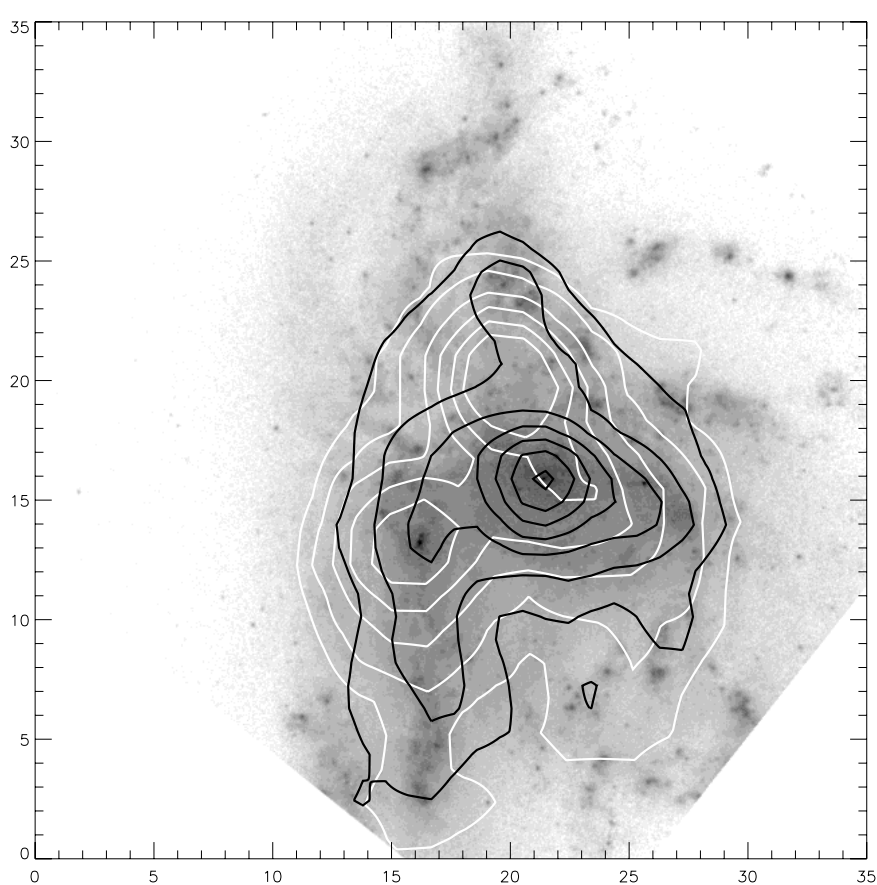

Fig. 3. An overlay of the ISO $15 \mu \mathrm{m}$ (white contours) and 2MASS $K$-band (black contours) on the HST image of the Mkn 297 galaxy. The HST image and ISO contours are as in Fig. 1. The 2MASS contours are equispaced at levels $350,355,360,365,370,375$ and 380 detector units. (We have not absolutely calibrated the 2MASS data, being only interested here in spatial information.)

highest for source regions $1,2,4$ and 3 respectively, with a peak value of 3.4 at source 1 and a global value of 1.5 to 2 over much of the galaxy.

\subsection{The PHT-S Spectrum}

The PHT-S spectrum is presented in Fig. 5, and line-fluxes derived for the features in the spectrum are given in Table 4. To allow for the limited (vignetted) aperture size of PHT-S with respect to the extent of the galaxy (see Fig. 1), the PHT-S signal was normalised to the ISOCAM 7.7 micron flux density as described in Sect. 2.4.

The most marked characteristic of the PHT-S spectrum is the presence of the family of infrared bands at 6.2, 7.7, 8.6 and $11.3 \mu \mathrm{m}$ that are generally attributed to emission from PAHs. There is a possible feature, seen at about the 3-sigma level, corresponding to the nebular line emission from [Ar III] at a restframe wavelength $8.99 \mu \mathrm{m}$. There is no convincing evidence of [S IV] at $10.51 \mu \mathrm{m}$. These features have been observed in other starburst galaxies (Metcalfe et al. 1996; Helou 1999; Laureijs et al. 2000; O'Halloran et al. 2000, 2002, 2005).

\subsection{The LWS spectrum}

The LWS line fluxes for the far-infrared lines at $157.7 \mu \mathrm{m}$ [CII], $88.8 \mu \mathrm{m}[\mathrm{O}$ II] and for [OI] at $63.1 \mu \mathrm{m}$ and $146.7 \mu \mathrm{m}$ are listed in Table 5 and corresponding spectra are presented in Fig. 6.

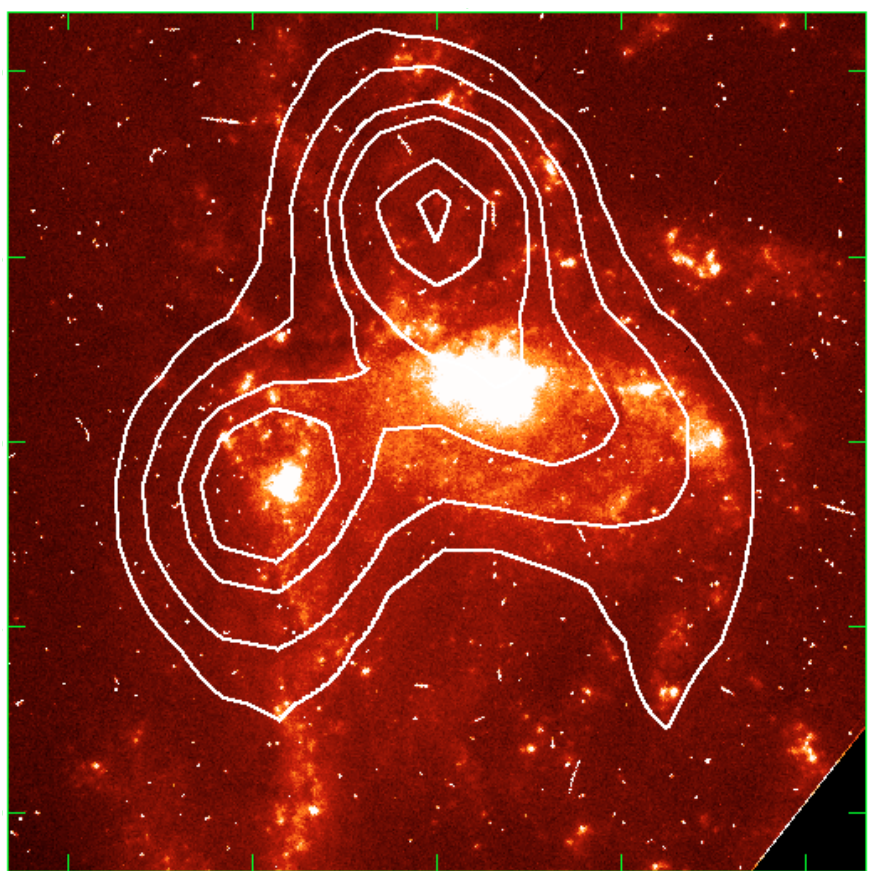

Fig. 4. The 14.3/6.7 um ratio map overlaid on the HST image of Mkn 297. Deconvolved 6.7 and $14.3 \mu \mathrm{m}$ maps were used and the ratio map was convolved with the $6.7 \mu \mathrm{m}$ point spread function. The ratio contour levels in ascending order are: 1.0, 1.5, 2.0, 2.5, 3.0, 3.3.

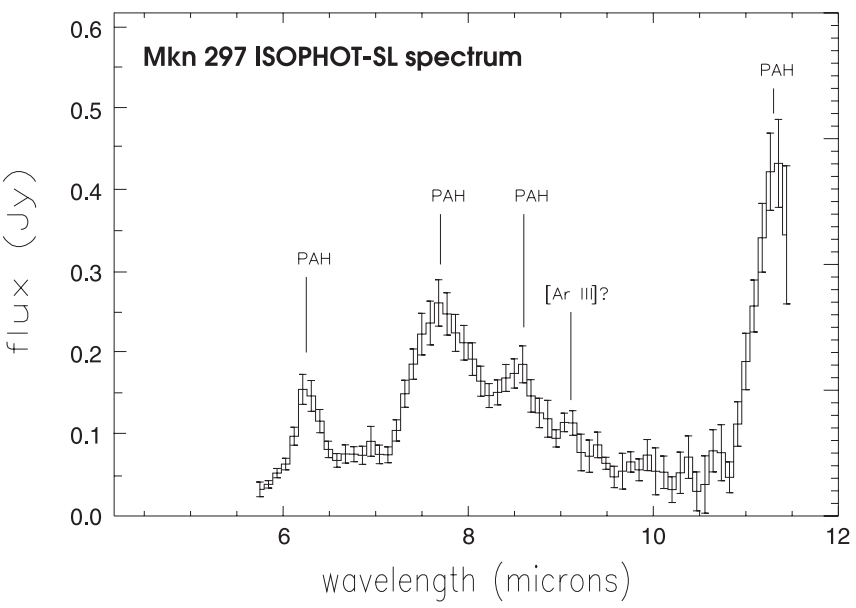

Fig. 5. PHT-SL spectrum of Mkn 297, with the PHT-S aperture centred $3^{\prime \prime}$ East of the nucleus of galaxy A. The PAH features are indicated. The spectrum has been Hanning smoothed. The flux levels are those recorded in the PHT-S aperture, before scaling to the larger ISOCAM field-of-view (see Sect. 2.4).

Table 6 lists the continuum source strength recorded in the ten LWS detectors by binning data across the spectral range of each detector. The spectral energy distribution of Mkn 297 using ISOCAM, PHT-SL, LWS and IRAS fluxes is presented in Fig. 7. 
Table 4. PHT-SL fluxes for Mkn 297. The columns give, respectively, the line identification, the wavelength range, and the integrated line-flux and the integrated line-flux scaled to the full ISOCAM footprint, as described in Sect. 2.4.

\begin{tabular}{lccc}
\hline \hline $\begin{array}{l}\text { Line ID } \\
(\mu \mathrm{m})\end{array}$ & $\begin{array}{c}\text { Wavelength range } \\
(\mu \mathrm{m})\end{array}$ & $\begin{array}{c}\text { Flux } \\
\left(\times 10^{-15} \mathrm{~W} / \mathrm{m}^{2}\right)\end{array}$ & $\begin{array}{c}\text { Flux } \\
\left(\times 10^{-15} \mathrm{~W} / \mathrm{m}^{2}\right)\end{array}$ \\
\hline PAH 6.2 & $5.8-6.6$ & $3.0 \pm 0.8$ & $4.4 \pm 1.2$ \\
PAH 7.7 & $7.2-8.2$ & $9.2 \pm 2.4$ & $13.4 \pm 3.6$ \\
PAH 8.6 & $8.3-8.9$ & $2.7 \pm 0.6$ & $3.9 \pm 0.9$ \\
PAH 11.3 & $10.9-11.6$ & $1.1 \pm 0.3$ & $1.7 \pm 0.5$ \\
\hline
\end{tabular}

Table 5. LWS line fluxes for Mkn 297. The three columns give the line identification, the wavelength and the line-flux respectively.

\begin{tabular}{lcc}
\hline \hline Line & $\begin{array}{c}\lambda \\
(\mu \mathrm{m})\end{array}$ & $\begin{array}{c}\text { Line-flux } \\
\left(\times 10^{-15} \mathrm{~W} / \mathrm{m}^{2}\right)\end{array}$ \\
\hline$[\mathrm{O}$ I] & 63.1 & $2.0 \pm 0.5$ \\
{$[\mathrm{O} \mathrm{III}]$} & 88.8 & $1.8 \pm 0.4$ \\
{$[\mathrm{O}$ I] } & 146.7 & $0.17 \pm 0.07$ \\
{$[\mathrm{C} \mathrm{II}]$} & 157.7 & $1.8 \pm 0.1$ \\
\hline
\end{tabular}
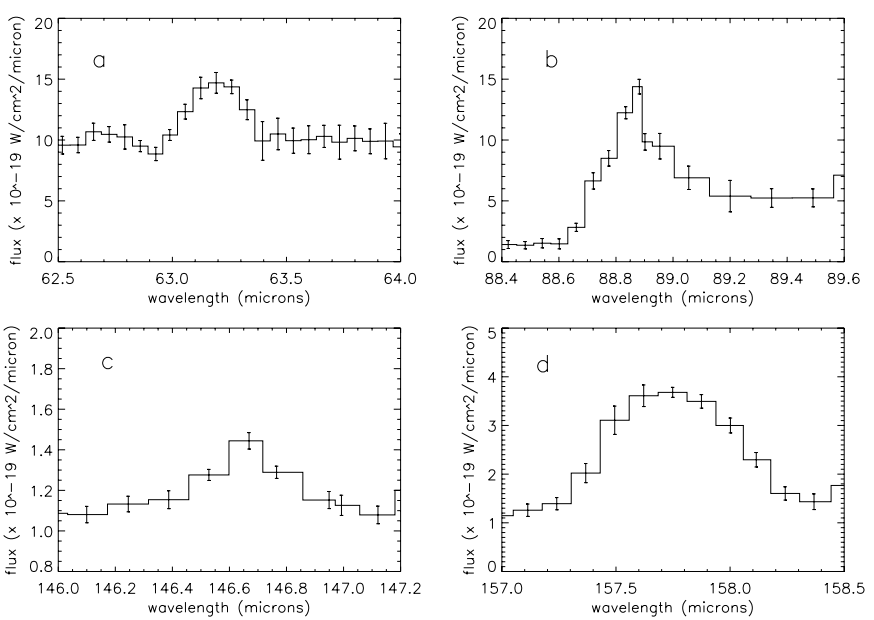

Fig. 6. LWS spectra of a) the [OI] line at $63.1 \mu \mathrm{m}$, b) the [OIII] line at $88.8 \mu \mathrm{m}$ (top right), c) the [OI] line at $146.7 \mu \mathrm{m}$ and d) the [CII] at $157.7 \mu \mathrm{m}$.

\section{Discussion}

\subsection{Model of the interacting system}

Taniguchi \& Noguchi (1991), using numerical $N$ body simulations, were able to account for the morphology and velocity structure of Mkn 297. The simulations suggested that a coplanar radial penetration between two disk galaxies yielded the wing formation which is seen now, about $1.5 \times 10^{8}$ years after the collision, when the disk of an edge-on (from the Earth's perspective) galaxy has been deformed into a wing structure. ISOCAM source 2 (Fig. 1) corresponds to the nucleus of the edge-on galaxy, and the North-South elongated structure corresponds to poorly formed tails emerging from the nucleus of this galaxy (Taniguchi \& Noguchi 1991). One of the best examples of a galaxy exhibiting such a tail morphology is the Antennae
Table 6. Flux densities measured with LWS, binned to yield a single number per LWS detector.

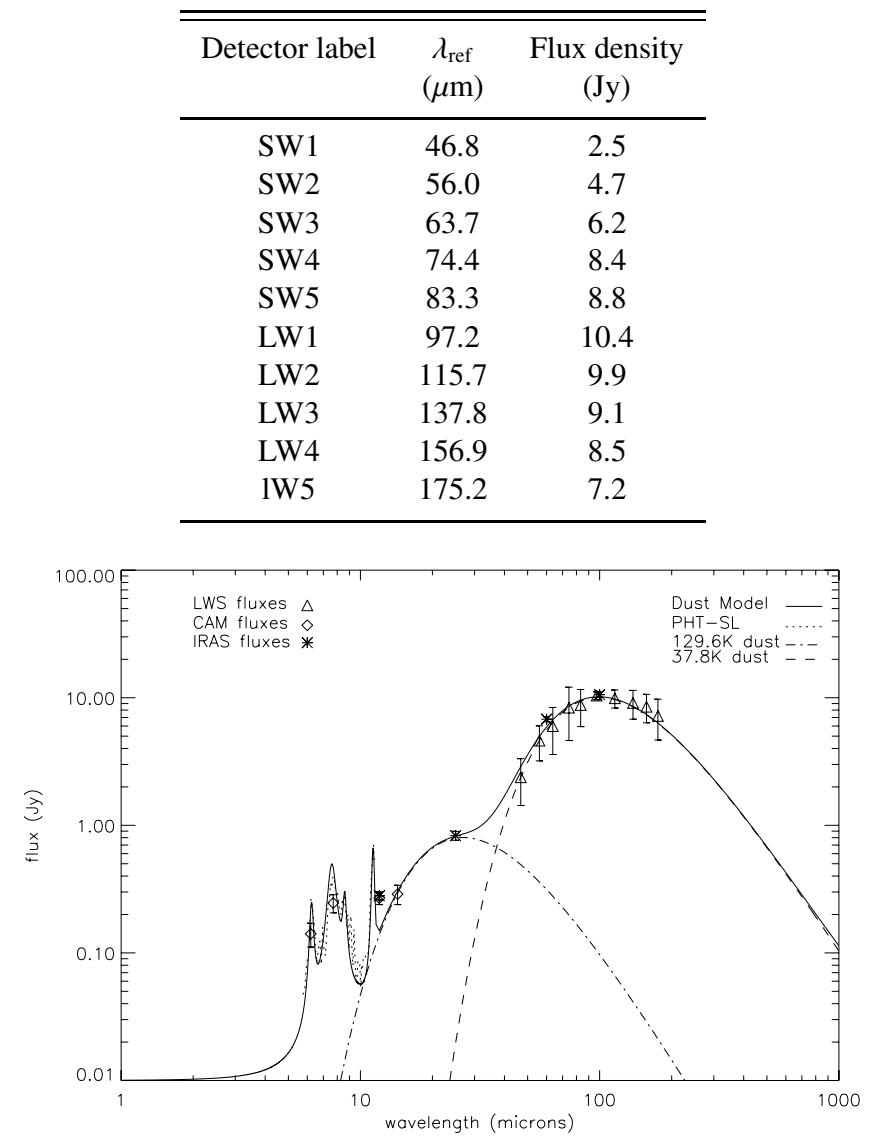

Fig. 7. Spectral energy distribution of Mkn 297 using ISO and IRAS flux data, including the key for the different symbols. The PHT-S spectrum has been normalised to the CAM $7.7 \mu \mathrm{m}$ data point as described in the text. The models fitted to the data are described in Sect. 4.4.

(NGC 4038/4039), where the two well developed tails are considered tidal debris from each component galaxy. The tail of Mkn 297 differs from the Antennae because it is not so well developed and is more luminous in the infrared, with a high $14.3 / 6.7 \mu \mathrm{m}$ ratio indicative of massive star formation.

The future evolution of the two galaxies has been predicted based on the simulations. After the wing phase, the stellar disk is deformed into a bar-like structure, gas clouds tend to sink into the nuclear regions of the edge-on galaxy and the nuclear starburst becomes dominant. It is expected that Mkn 297 will 
evolve into two galaxies, one a starburst nucleus galaxy and the other a ring galaxy. (See also Efstathiou et al. 2000.)

The simulations also suggest a separation between the two galaxies of about four galactic radii, and hence the orbital plane of the collision must be significantly inclined from the plane of the sky (by about 70 degrees, to account for the apparent closeness of the two galactic nuclei).

\subsection{HII and CO observations}

Mkn 297 has an optical spectrum typical of a HII region, with line intensities consistent with excitation by massive young stars. The nuclear region of galaxy A (source 4 in Fig. 1) has the highest optical surface brightness, with a strong stellar continuum and an abundance of $[\mathrm{O} / \mathrm{H}] \approx 8.7$, which is near the solar value and typical of late-type spiral galaxies (Lonsdale et al. 1992). The nuclear region of galaxy B (source 2 in Fig. 1) has a much bluer spectrum, with an excitation comparable to that of the HII regions in the Large Magellanic Cloud (Burenkov 1988).

Compact CO line emission from Mkn 297 has been mapped and, at the resolution of the systems used (of the order of $20^{\prime \prime} H P B W$ ) has been associated with the optical knots (Sage et al. 1993; Sofue et al. 1990). The estimated mass of $\mathrm{H}_{2}$ is $\sim 8 \times 10^{9} M_{\odot}$ and is approximately $75 \%$ of the $\mathrm{HI}$ mass of $1.1 \times 10^{10} M_{\odot}$.

\subsection{Far infrared spectral analysis}

Atomic oxygen and ionized carbon are the principal coolants of the gaseous interstellar medium via their fine structure lines in the far infrared (FIR). In particular, [OI] at $63 \mu \mathrm{m}$ and [CII] at $158 \mu \mathrm{m}$ dominate the cooling in the photodissociation regions associated with massive stars such as Wolf Rayets, along with [OI] at $146 \mu \mathrm{m}$ and [OIII] at $88 \mu \mathrm{m}$ (Sanders et al. 2003). The $[\mathrm{OI}]$ and $[\mathrm{CII}]$ features are also produced in the warm atomic gas behind dissociative shocks, in HII regions or in photodissociation regions (PDRs), while [OIII] is more associated with denser environments within HII regions (Malhotra et al. 1997; Braine \& Hughes 1999).

The [OI] $63 \mu \mathrm{m}$ and [CII] $158 \mu \mathrm{m}$ lines were well detected in Mkn 297 and were used to determine the luminosity ratios $L_{\mathrm{CII}} / L_{\mathrm{FIR}}$ and $\left(L_{\mathrm{CII}}+L_{\mathrm{OI}}\right) / L_{\mathrm{FIR}}$, which probe the nature of the environment within the galaxy. Values of $L_{\mathrm{CII}} / L_{\mathrm{FIR}}=5.1 \times 10^{-3}$ and $\left(L_{\mathrm{CII}}+L_{\mathrm{OI}}\right) / L_{\mathrm{FIR}}=10.7 \times 10^{-3}$ were determined using the IRAS flux-densities (Malhotra et al. 1997). The values found are consistent with those from other starburst galaxies (Malhotra et al. 1997; Braine \& Hughes 1999), though for higher dust temperatures and star formation rates these ratios decrease (Malhotra et al. 1997). Mkn 297 falls at the higher star formation/dust temperature end of this correlation, which provides further evidence of strong ongoing star formation within this galaxy.

The [CII] line emission is the dominant gas coolant in most regions of atomic interstellar gas and therefore reflects the heating input to the gas (Malhotra et al. 2001). The ratio of [CII]/far-infrared (FIR), as a function of the ratio of the flux density at $60 \mu \mathrm{mm}$ to $100 \mu \mathrm{m}, R(60 / 100)$, has been measured for a large sample of galaxies and used to study the radiation field (Helou et al. 2001). The [CII]/FIR ratio for Mkn 297 is $0.51 \times 10^{-2}$ and $R(60 / 100)$ is 0.64 and these values are typical of a star forming galaxy as shown in Fig. 1 in Helou et al. (2001). A strong correlation was also found between [CII] and the integrated mid-infrared flux in the range $5-10 \mu \mathrm{m}$ in a large sample of star forming galaxies. The mid-infrared flux is dominated by aromatic features (AFEs), that are generally associated with the smallest interstellar grains. The ratio of the two quantities [CII]/AFE, where AFE is $v f(v)$ over the wavelength range $5-10 \mu \mathrm{m}$, is nearly constant at $1.5 \%$ over a wide range in values of $R(60 / 100)$ (Helou et al. 2001; Dale et al. 2000). In Mkn 297, the value of $v f(v)$ was synthesised from the PHT-SL spectrum (Fig. 5), extrapolating the continuum to 5 microns in the integral. The resulting value was scaled to the area of the ISOCAM footprint, as described in Sect. 2.4, resulting in a high value of $[\mathrm{CII}] / \mathrm{AFE}, 4 \%$. Although the value of $4 \%$ might be regarded as an upper limit because the PHT-SL measurement covered a much smaller field of view than the LWS spectrometer (Table 1). However, as argued in Sect. 2.4, it is unlikely that much flux in the PHT-SL range falls outside of the ISOCAM footprint to which it has been scaled. The value of $4 \%$ is an additional argument in favour of star formation dominating the mid-infrared emission.

The unidentified infrared bands (UIBs) dominate the midinfrared emission from Mkn 297 (Fig. 5) with little or no emission in the wavelength region between 9 and $10 \mu \mathrm{m}$ from very small grains (VSGs). This result is also observed in IC 694 (Source A in Arp 299) but not in knot A in the Antennae. In cases where the mid-infrared emission is dominated by UIBs, the SFR can be obtained from the following relationship (Roussel et al. 2001):

$$
\operatorname{SFR}\left(M_{\odot} \mathrm{yr}^{-1}\right)=6.5 \times 10^{-9} L(15 \mu) .
$$

The flux density at $15 \mu \mathrm{m}$ was converted into luminosity using a bandpass of $6.75 \mathrm{THz}$. This yields SFR in Mkn 297 of $17.1 M_{\odot} \mathrm{yr}^{-1}$. Taniguchi \& Noguchi (1991) state a FIR-based value of $23.5 M_{\odot} \mathrm{yr}^{-1}$ for the SFR in Mkn 297.

The SFR can also be obtained from the $\mathrm{H}_{\alpha}$ luminosity using (Lee et al. 2002):

$\operatorname{SFR}\left(M_{\odot} \mathrm{yr}^{-1}\right)=7.9 \times 10^{-42} L\left(\mathrm{H}_{\alpha}\right) \mathrm{erg} \mathrm{s}^{-1}$.

The $\mathrm{H}_{\alpha}$ flux from Mkn 297 is $21 \times 10^{-13} \mathrm{erg} \mathrm{s}^{-1} \mathrm{~cm}^{-2}$ (Deeg et al. 1997) which yields a value of $8.6 M_{\odot} \mathrm{yr}^{-1}$. These results suggests that about half of the star formation in Mkn 297 is hidden in the optical.

\subsection{Dust components within Mkn 297 and the FIR-radio correlation}

A dust model for Mkn 297 is shown in Fig. 7, denoted by the solid curve. It includes two separate dust populations: a warm dust component at $130 \mathrm{~K}$ and a cooler dust component at $38 \mathrm{~K}$ (Krugel \& Siebenmorgen 1994; Siebenmorgen et al. 1999).

There is growing evidence for the existence of several components in the dust distribution of galaxies (Klaas et al. 2001; 
Table 7. Total infrared luminosity and dust component masses for Mkn 297. The total FIR luminosity of $9.7 \times 10^{10} L_{\odot}$ places this galaxy right on the threshold of LIRG status.

\begin{tabular}{lcc}
\hline \hline Dust component & $\begin{array}{c}L_{\mathrm{IR}} \\
{\left[L_{\odot}\right]}\end{array}$ & $\begin{array}{l}\text { Mass } \\
{\left[M_{\odot}\right]}\end{array}$ \\
\hline $130 \mathrm{~K}$ & $7.5 \pm 0.8 \times 10^{9}$ & $4.2 \pm 0.4 \times 10^{3}$ \\
$38 \mathrm{~K}$ & $8.9 \pm 0.9 \times 10^{10}$ & $3.3 \pm 0.4 \times 10^{7}$ \\
\hline
\end{tabular}

Popescu et al. 2002) that can be broken down into warm dust components with $T \geq 20 \mathrm{~K}$ associated with star formation regions, and a spatially extended distribution of cold dust with $T \leq 20 \mathrm{~K}$.

Very small dust grains and PAHs are transiently heated by the single-photon emission process to temperatures much higher than $40 \mathrm{~K}$, up to several hundred degrees Kelvin (Désert et al. 1990), out of thermal equilibrium with their environment (Calzetti et al. 2000). These account for the bulk of the emission in the PHT-S waveband and for emission at wavelengths shorter than $40 \mu \mathrm{m}$. The "warmer" or "hot dust" component, the $130 \mathrm{~K}$ component in Fig. 7, is due to small grains.

Dust emission from HII regions is dominated by large dust grains heated by the intense radiation field. These grains can reach temperatures in excess of $20 \mathrm{~K}$ up to more than $40 \mathrm{~K}$. Due to the nature of the blackbody, this emission will outshine the cold dust in these regions. The $38 \mathrm{~K}$ dust component in Fig. 7 comes from large grains in HII regions.

The coldest dust emission is associated with large grains emitting at wavelengths in excess of $80 \mu \mathrm{m}$ and in thermal equilibrium with their environment (Calzetti et al. 2000). A small contribution at $100 \mu \mathrm{m}$ is also made by very small dust grains. The large dust grains account for practically all the emission longward of $80 \mu \mathrm{m}$ in galaxies, with the maximum of the SED occurring close to $200 \mu \mathrm{m}$. We have not detected this very cold component in Mkn 297 within the sensitivity of the current measurements (and given the occurrence of cirrus and zodiacal light confusion at the longest wavelengths).

The luminosity of each component was found by integrating over a model greybody, fitting temperatures and calculating a dust mass for the component (Klaas \& Elsaesser 1991). Using this method, the IR luminosities and dust component masses in similar starburst galaxies such as the Antennae and NGC 6240 (Klaas et al. 1997) were calculated in order to check the validity of the derived values. The values obtained were generally in good agreement The dust model yields for Mkn 297 a total $L_{\mathrm{IR}}$ of $9.7 \times 10^{10} L_{\odot}$. For each component, the luminosities and dust masses are given in Table 7 . The warmer dust emission is concentrated within the disk and associated with the strongly ionizing sources found in star forming regions.

Deeg et al. (1997) report radio data for a source within 3 " of Source 1 having flux densities of $244 \pm 20 \mathrm{mJy}$ and $104 \pm 7 \mathrm{mJy}$ at $0.325 \mathrm{GHz}$ and $1.489 \mathrm{GHz}$ respectively. This is consistent with the variable radio source reported by Heeschen et al. (1983), which varied at $4.885 \mathrm{GHz}(6 \mathrm{~cm})$ from $4.2 \mathrm{mJy}$ on January 121980 to $12.2 \mathrm{mJy}$ on April 23 of that year. See also Yin et al. (1994), and Fig. 8.

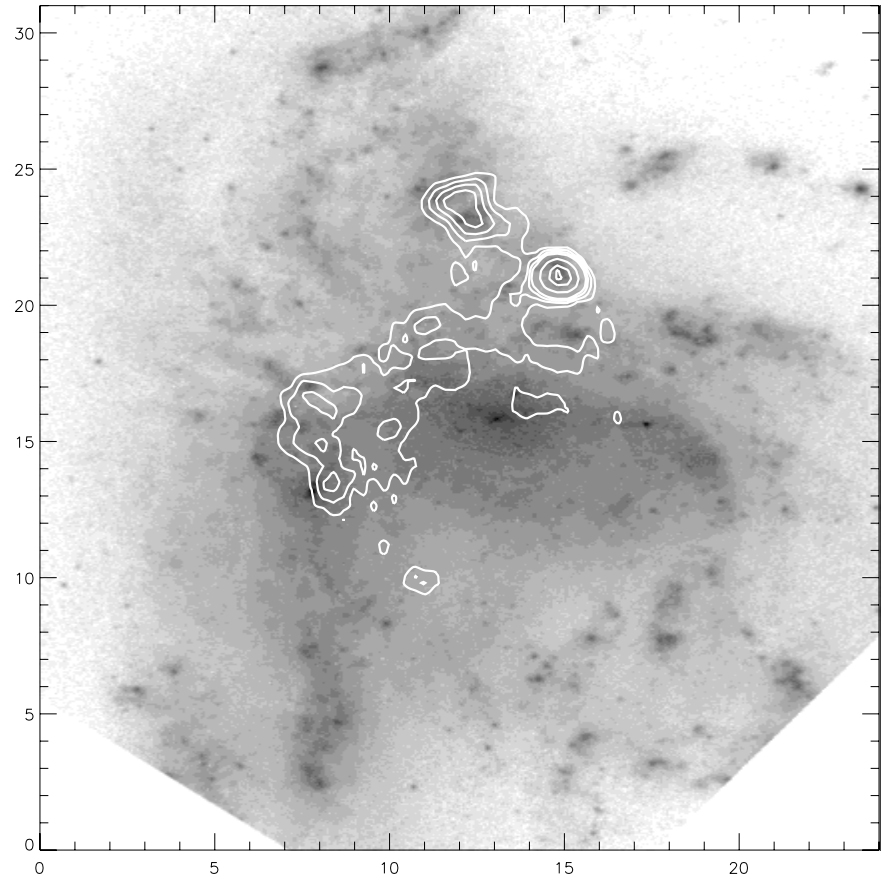

Fig. 8. An overlay of the Yin (1994) $6 \mathrm{~cm}$ radio contours on the HST image of Mkn 297.

Pierini et al. (2003) give expressions to relate warm and cold FIR emission to radio flux, and the values for FIR emission for Mkn 297 given in Table 7 fall on the correlations in each case. From Condon et al. $(1990), \log (L(1.4 \mathrm{Ghz}))=22.74$, while Table 7 yields $\log (L(\mathrm{FIR}))=37.85$. For the warm and cold dust respectively: $\log (L(\mathrm{FIR}))=36.74$ and $\log (L(\mathrm{FIR}))=37.82$. All lie close to the relevant correlations, though it must be remarked that the total FIR luminosity for Mkn 297, and particularly the cold component, fall towards the high FIR extreme of the Pierini et al. correlations.

\subsection{The hidden source in Mkn 297 and comparison with other cases}

The JHK 2MASS images of Mkn 297 reveal a source that is North of galaxy A and coincides with the star forming regions 7 and 8 of Hecquet et al. (1987). An overlay of the ISO $15 \mu \mathrm{m}$ map and the 2MASS $K$-band map on the HST image is shown in Fig. 3. The $K$-band peak clearly corresponds to the optically evident HII region, and not at all to the hidden MIR source Source 1 . The star forming region that is nearest to the hidden source is region 16 of Hecquet et al. (1987) and has the largest extinction of all the star forming regions in Mkn 297. The hidden source in Mkn 297 is inconspicuous in the optical and also in the JHK 2MASS images.

There are three other sources where strong mid-infrared emission has been detected that are unremarkable in the optical. The first source is knot A in the overlap region of the colliding galaxies in the Antennae (Vigroux et al. 1996; Mirabel et al. 1998), the second is in the intragroup region of Stephan's Quintet that could be an intruding galaxy (Xu et al. 1999) and 
Table 8. A comparison of the properties the hidden sources (h.s.) in four systems. Column 3 lists the total $15 \mu \mathrm{m}$ emission from the host galaxy in each case.

\begin{tabular}{lcccccc}
\hline \hline Source & $\begin{array}{c}7 \mu \mathrm{m} \\
\mathrm{mJy} \\
\text { (h.s.) }\end{array}$ & $\begin{array}{c}15 \mu \mathrm{m} \\
\mathrm{mJy} \\
\text { (h.s.) }\end{array}$ & $\begin{array}{c}15 \mu \mathrm{m} \\
\mathrm{mJy} \\
\text { (host) }\end{array}$ & $\begin{array}{c}D \\
\mathrm{Mpc}\end{array}$ & $R 1$ & $R 2$ \\
\hline Mkn 297 & 42.5 & 66.5 & 289 & 66 & - & 0.23 \\
Antennae & 19.1 & 49.7 & 261.7 & 20.0 & 14.6 & 0.19 \\
Stepn's Q. & - & 11.9 & 114.9 & 80 & 3.8 & 0.10 \\
Arp 299 & 325 & 1860 & 7027 & 41 & 0.09 & 0.26 \\
\hline
\end{tabular}

$R 1=$ Ratio of $15 \mu \mathrm{m}$ (LW3) emission from the hidden

source in Mkn 297 to the hidden source in the other galaxy (corrected for distance to Mkn 297).

$R 2=$ Ratio of $15 \mu \mathrm{m}$ emission from each hidden source to the total $15 \mu \mathrm{m}$ emission for its host galaxy.

the third is source A or IC694 in the interacting galaxies in Arp 299 (Charmandaris et al. 2002; Gallais et al. 2004). The hidden source in Mkn 297 is important in this context and is very similar to those in the Antennae and Stephan's Quintet because the hidden sources do not coincide with the nuclei of any of the interacting galaxies.

At $15 \mu \mathrm{m}$ (LW3), the hidden source in Mkn 297 is 14.6 times more luminous than knot $\mathrm{A}$ in the Antennae, and 3.8 times more luminous than the hidden source in Stephan's Quintet. However, its luminosity is only 0.09 times that of the hidden source IC 694 in Arp 299. However the ratio of the LW3 flux from the hidden source to the total flux is similar for all sources with values of $0.23,0.19,0.26$ and 0.10 for Mkn 297, the Antennae, Arp 299 and Stephan's Quintet respectively.

The hidden source in Mkn 297 appears to be more similar in properties to knot $\mathrm{A}$ in the Antennae, than it is to the nuclear source IC 694 (Charmandaris et al. 2002; Gallais et al. 2004). The source in IC 694 becomes visible as a point source in the $J H K$ 2MASS images and gets brighter from $J$ to $H$ to $K$ and also contributes significantly to the near infrared radiation from the galaxy. However this is not the case in Mkn 297 or the Antennae, where knot $\mathrm{A}$ is inconspicuous in the near infrared and becomes dominant in the mid-infrared. Table 8 lists comparative infrared properties for Mkn 297 and three sources with somewhat similar morphological characteristics.

The differences between the hidden sources suggest that dust extinction is lower in the hidden source in IC 694 than in Mkn 297 or knot A in the Antennae. An alternative interpretation is that the thermal emission from an enshrouded AGN becomes visible in the near infrared. This phenomenon was observed in the nucleus of NGC 1068 (Alonso-Herrero et al. 2003) and will be discussed further in Sect. 4.6. The variable and compact radio source Mkn 297A (Fig. 8) that falls on the edge of the hidden source in Fig. 1 has been studied extensively (Hummel et al. 1987; Condon et al. 1991; Yin \& Heeschen 1991; Yin 1994). This source was unresolved with the resolutions of the VLA and the flux density shows a rapid rise followed by a slower decline with maximum flux density occurring first at the shortest wavelength (Yin 1994). The flux density, $S$, was modelled as a power law function of both frequency and time of the form

$S \propto v^{\alpha} t^{\beta}$

which is characteristic of radio supernovae. The values of the indices $\alpha$ and $\beta$ for the variable radio source are typical of radio supernovae. These results reveal that the radio source close to the hidden source is a radio supernova and not a dominant AGN.

The VLA map of Mkn 297 (Fig. 8) shows that the variable radio source coincides with star forming region 14 of Hecquet et al. (1987).

\subsection{Does Mkn 297 harbour an AGN?}

Several diagnostic tools have been proposed to probe the nature of the activity within a central starburst region. Lu et al. (1999) cite the ratio of the integrated PAH luminosity and the 40 to $120 \mu \mathrm{m}$ IR luminosity as providing a tool to discriminate between starbursts, AGN and normal galaxies. The lower the ratio the more likely the galaxy harbours an AGN, due to the dominance of very small dust grain emission powered by the AGN in the 40 to $120 \mu \mathrm{m}$ region (Vigroux et al. 1999). For Mkn 297, the ratio is 0.11, which is typical of a strong starburst. Similarly, Lutz et al. (1998), Genzel et al. (1998), Laureijs et al. (2000) state that the ratio of the $7.7 \mu \mathrm{m}$ PAH flux to the nearby continuum can provide a measure of the level of star formation activity within the nucleus, given the ubiquity of strong PAH features in regions of high star formation (Clavel et al. 2000). The ratio for Mkn 297 is 3.7. Plotting this value, and the $5.9 \mu \mathrm{m} / 60 \mu \mathrm{m}$ flux densities $(0.061 \mathrm{Jy}$ in the PHT-S aperture/6.79Jy from IRAS) in Fig. 3 of Laureijs et al. (2000) indicates strong star formation, suggesting that an AGN within Mkn 297 is unlikely (O'Halloran et al. 2000; Laureijs et al. 2000).

Another set of diagnostics uses empirical criteria based on the fact that mid-infrared emission from star forming or active galaxies arises mostly from HII regions, photo-dissociation regions (PDRs) and AGNs (Laurent et al. 2000). The diagnostic diagrams provide quantitative estimates of the contributions of AGN, PDR and HII regions. These diagrams are referred to as the Laurent diagnostic diagrams (Peeters et al. 2004). In Mkn 297 the ratio of LW3/LW2 is 2 and that of LW2/LW4 is roughly 2, where the LW4 value was synthesised from the PHTSL spectrum, making approximate allowance for the fact that the LW4 bandpass extends to shorter wavelength than PHT-S. These values show that the dominant contribution to the midinfrared emission is from PDR and HII regions in Mkn 297 and not from an AGN.

However, mid-IR diagnostics cannot probe beyond values of about 10 magnitudes of extinction, and so miss any AGN embedded deep into a star forming region with, say, 100 magnitudes of extinction. The absence of an AGN within Mkn 297 can therefore only be tentatively suggested until it is confirmed by hard X-ray observations. 


\subsection{Unusual radio supernova remnant and possible SN/GRB link}

Heeschen et al. (1983) found a peculiar, compact and variable radio source which falls about $2 \mathrm{kpc}$ north of the nucleus of galaxy A, on the edge of the strongest infrared source in Fig. 1 (source 1). They suggested, among other possibilities, that it might be a complex of supernova remnants. In subsequent radio VLA observations (Yin \& Heeschen 1991; Yin 1994) a decay in flux density from $14 \mathrm{mJy}$ to $4.7 \mathrm{mJy}$ at $20 \mathrm{~cm}$ was recorded between 1983 and 1990, and the source (Fig. 8) was attributed to a single, very luminous, radio supernova that exploded in mid-1979. The radio SN was later cataloged as SN 1982aa, of uncertain type because of the paucity of radio results within its first 8 years and the absence of optical observations (Green 1994). VLBI observations (Lonsdale et al. 1992) are consistent with the single supernova occurring in a molecular cloud.

The most surprising aspect of the supernova interpretation was the very high radio luminosity of $\sim 1.5 \times 10^{5} \mathrm{~L}_{\odot}$. As a radio supernova it is one of the most energetic events ever observed, alongside SN 1986 J, 41.9+58 in M82, SN 1998bw and SN 2003dh (Wilkinson \& de Bruyn 1990; Galama et al. 1998; Berger et al. 2003). These powerful supernovae are more energetic, by a factor of 10 , than canonical supernovae which release $\geq 10^{52} \mathrm{erg}$ during the explosion. Indeed, SN 1982aa has a total released energy of $10^{53 \pm 0.5} \mathrm{erg}$, as derived from the radio flux reported by (Yin 1994).

So far, many of the most powerful supernovae have been of type $\mathrm{Ib} / \mathrm{c}$, the best known example of which has been SN 1998bw, one of the most luminous radio SN ever observed (Weiler et al. 2001, 2002). Interestingly, SN 1998bw has been strongly associated with a weak gamma-ray burst (GRB), GRB 980425 (Galama et al. 1998; Kouveliotou et al. 2004; Soderberg et al. 2004). It has only been exceeded in radio luminosity by SN 1982aa (Yin 1994) and SN 2003dh, which is identified with the cosmological burst GRB 030329 (Berger et al. 2003).

A well known model for the progenitors of GRBs, linked to powerful supernovae, and by extension to regions of massive star formation, is the "collapsar" model. In this model the core of a massive star collapses to a black hole, which then powers the GRB by the accretion of an additional solar mass (McFadyean \& Woosley 1999), and signatures consistent with spin-up and spin-down of black holes have been found in the light profiles of GRBs (McBreen et al. 2002). For SN 1982aa, given the amount of energy released it is quite plausible that a GRB may have been associated with this candidate obscured supernova. Furthermore, Mkn 297 is morphologically similar to host galaxies of GRBs seen in HST images, which show most GRB host galaxies to be either interacting or disturbed (Wainwright 2005). In 1979, many spacecraft had GRB detectors and a search is recommended in data from the period July through December 1979, for a GRB consistent with the direction to Mkn 297. It is also important to continue radio observations of Mkn 297A so that a proper comparison can be made of its long term radio profile with that of SN 1998bw, SN 2003dh and other supernovae (Frail et al. 2000) with associated GRBs that have yet to be discovered.

\section{Conclusions}

Mkn 297 is an interacting system possibly involving a latetype spiral and an irregular galaxy or two disk galaxies. It has a "wing" like structure extending North and South from the nuclear region. It was observed with ISOCAM, ISOPHOT and LWS on board ISO. The results obtained with the four ISOCAM filters used revealed a complex mid-infrared emission pattern with many clumps occurring within an overall envelope. The two nuclear regions and the wing structure were easily detected in all ISOCAM bandpasses employed. However the strongest infrared source does not coincide with either of the nuclei of the interacting galaxies but is located several arcseconds to the North, nor is that source seen in NIR (e.g. 2MASS) images. Strong PAH features were detected in the ISOPHOT spectrum, while [OI], [O III] and [C II] emission lines were detected with the LWS instrument. Using fluxes from these 3 instruments, masses and luminosities were determined for two dust components within Mkn 297. The total infrared luminosity of $9.7 \times 10^{10} L_{\odot}$ classifies it as a borderline LIRG. Numerous criteria discriminating star formation from AGN activity point to the energy source in Mkn 297 being star formation, and establish a stronger similarity between Mkn 297 and the Antennae system (NGC 4038/4039) rather than, for example, with less deeply obscured interaction-driven starbursts in IC 694 or Stephan's Quintet. Only hard X-ray measurement, however, can definitively eliminate the possibility that Mkn 297 harbours a highly obscured AGN. One of the strongest known radio supernovae, SN 1982aa, falls in a HII region impinging on the brightest infrared source in Mkn 297, and has been labeled a radio hypernova, calling to mind the suggested association between intensely star-forming systems (LIRGS and ULIRGS) and GRBs. Further observations of this radio supernova are recommended, as well as a search for a gamma ray burst around the time of the explosion in 1979.

Acknowledgements. We would like to thank the referee, Dr. Vassilis Charmandaris, for detailed comments which greatly improved the paper. We gratefully thank Dr. Qi Feng Yin for providing us with his VLA radio maps for study and for the radio data reproduced in Fig. 8. We thank Dr. Avishay Gal-Yam for helpful comments ans suggestions. The ISOCAM data presented in this paper were analysed using CIA, a joint development by the ESA Astrophysics Division and the ISOCAM Consortium. The ISOCAM Consortium is led by the ISOCAM PI, C. Cesarsky. The ISOPHOT data presented in this paper was reduced using PIA, which is a joint development by the ESA Astrophysics Division and the ISOPHOT consortium. LIA is a joint development of the ISO-LWS Instrument Team at Rutherford Appleton Laboratories and the Infrared Processing and Analysis Center (IPAC). The research of M. Burgdorf was carried out at the Jet Propulsion Laboratory, California Institute of Technology, and sponsored by the National Aeronautics and Space Administration. This publication makes use of data products from the Two Micron All Sky Survey, which is a joint project of the University of Massachusetts and the Infrared Processing and Analysis Center/California Institute of Technology, funded by the National Aeronautics and Space Administration and the National Science Foundation. 


\section{References}

Abergel, A., Miville-Deschênes, M. A., Désert, F. X., et al. 2000, ExA, 10,353

Acosta-Pulido, J. A., Gabriel, C., \& Castañeda, H. 2000, Exp. Astron., 10, 333

Alloin, D., \& Duflot, R. 1979, A\&A, 78, L5

Alonso-Herrero, A., Quillen, A. C., Rieke, G. H., et al. 2003, AJ, 126, 81

Berger, E., Kulkarni, S. R., Frail, D. A., \& Soderberg, A. M. 2003, ApJ, 599, 408

Blommaert, J., Siebenmorgen, R., Coulais, A., et al. 2003, The ISO Handbook, Volume II: CAM - The ISO Camera, ESA SP-1262, SAI-99-069/Dc, Version 2.0

http://www.iso.vilspa.esa.es/users/handbook/

Braine, J., \& Hughes, D. H. 1999, A\&A, 344, 779

Burenkov, A. N. 1988, Astrofizika, 28, 47

Cairós, L. M. Caon, N., Papaderos, P., Noeske, K., et al. 2003, ApJ, 593, 312

Calzetti, D., Armus, L., Bohlin, R. C., et al. 2000, ApJ, 533, 682

Cesarsky, C., Abergel, A., Agnese, P., et al. 1996, A\&A, 315, L32

Cesarsky, C., \& Sauvage, M. 1999, Ap\&SS, 269, 303

Charmandaris, V., Laurent, O., Mirabel, I. F., et al. 1999, Ap\&SS, 266, 99

Charmandaris, V., Stacy, G. J., \& Gull, G. 2002, ApJ, 571, 282

Clavel, J., Schulz, B., Altieri, B., et al. 2000, A\&A, 357, 839

Clegg, P. E., Ade, P. A. R., Armand, C., et al. 1996, A\&A, 315, 38

Condon, J. J., Helou, G., Sanders, D. B., \& Soifer, B. T. 1990, ApJS, 73,359

Condon, J. J., Frayer, D. T., \& Broderick, J. J. 1991, AJ, 101, 362

Dale, D. A., Silbermann, N. A., Helou, G., et al. 2000, ApJ, 120, 583

Deeg, H. J., Brinks, E., Duric, N., et al. 1993, ApJ, 410, 626

Deeg, H. J., Duric, N., Brinks, E., et al. 1997, A\&A, 323, 323

Delaney, M., \& Ott, S. 2002, http://www.iso.vilspa.esa.es/ users/expl_lib/CAM_top.html, ISOCAM Interactive Analysis User's Manual, v5.0, SAI/96-5226/Dc.

Désert, F., Boulanger, F., \& Puget, J. L. 1990, A\&A, 237, 215

Duflot, R. 1976, A\&A, 48, 437

Efstathiou, A., Rowan-Robinson, M., \& Siebenmorgen, R. 2000, MNRAS, 313, 734

Frail, D. A., Waxman, E., \& Kulkarni, S. R. 2000, ApJ, 537, 191

Gabriel, C. 2002, PHT Interactive Analysis User Manual, ESA, http://www.iso.vilspa.esa.es/manuals/PHT/ pia/pia.html

Galama, T. J., Vreeswijk, P. M., van Paradijs, J., et al. 1998, Nature, 395, 670

Gallais, P., Charmandaris, V., Le Floc'h, E., et al. 2004, A\&A, 414, 845

Genzel, R., Lutz, D., Sturm, E., et al. 1998, ApJ, 498, 579

Green, D. E. 1994, IAU Circular, 5953, 3

Gry, C., Swinyard, B. M., Harwood, A., et al. 2003, ISO Handbook, Volume III: LWS - The Long-Wavelength Spectrometer, ESA SP-1262, SAI-99-069/Dc, Version 2.1 http://www.iso.vilspa.esa.es/users/handbook/

Hecquet, J., Coupinot, G., \& Maucherat, A. J. 1987, A\&A, 183, 13

Heeschen, D. S., Heidmann, J., \& Yin, Q. F. 1983, ApJ, 267, L73

Helou, G., Malhotra, S., Hollenbach, et al. 2001, ApJ, 548, L73

Helou, G. 1999, in The Universe as seen by ISO, ed. P. Cox, \& M.F. Kessler, ESA SP-427, 2, 797

Hensler, G., Dickow, R., \& Junkes, N. 1997, in Rev. Mex. Astron. Astrofis. Ser. Conf., 6, 90

Hummel, E., van der Hulst, J. M., Keel, W. C., et al. 1987, A\&AS, 70, 517
Kessler, M. F., Steinz, J. A., Anderegg, M. E., et al. 1996, A\&A, 315, L27

Klaas, U., \& Elsaesser, H. 1991, A\&AS, 90, 33

Klaas, U., Haas, M., Heinrichsen, I., \& Schulz, B. 1997, A\&A, 325, L21

Klaas, U., Haas, M., Muller, S. A. H., et al. 2001, A\&A, 379, 823

Kouveliotou, C., Woosley, S. E., Patel, S. K., et al. 2004, ApJ, 608, 872

Krugel, E., \& Siebenmorgen, R. 1994, A\&A, 282, 407

Kunze, D., Rigopoulou, D., Lutz, D., et al. 1996, A\&A, 315, L101

Laureijs, R., Watson, D., Metcalfe, L., et al. 2000, A\&A, 359, 900

Laureijs, R. J., Klaas, U., Richards, P. J., Schulz, B., \& Ábrahám, P. 2003, The ISO Handbook, Volume IV: PHT - The Imaging Photo-Polarimeter, ESA SP-1262, SAI-99-069/Dc, Version 2.0.1 http://www.iso.vilspa.esa.es/users/handbook/

Laurent, O., Mirabel, L. F., Charmandaris, V., et al. 2000, A\&A, 359, 887

Lee, J., Salzer, J., Impey, C., Thuan, T. X., \& Gronwall, C. 2002, AJ, 124, 3088

Lemke, D., Klaas, U., Abolins, J., et al. 1996, A\&A, 315, L64

Lloyd, C., Lerate, M.R., \& Grundy, T.W. 2003, Uniformly processed LWS L01 spectra, The LWS LO1 Pipeline Version 1. http://pma.iso.vilspa.esa.es: $8080 / \mathrm{hpdp} /$ technical_reports/technote17.html

Lonsdale, C. J., Lonsdale, C. J., \& Smith, H. E. 1992, ApJ, 391, 629

Lu, N. Y., Helou, G., Silbermann, N. et al. 1999, in The Universe as seen by ISO, ed. P. Cox, M. F. Kessler, ESA SP-427, 2, 929

Lutz, D., Spoon, H. W. W., Rigopoulou, D., Moorwood, A. F. M., \& Genzel, R. 1998, ApJ, 505, L103

Malhotra, S., Helou, G., Stacey, G., et al. 1997, ApJ, 491, 27

Malhotra, S., Kaufman, M. J., Hollenbach, D. J., et al. 2001, ApJ, 561, 766

McBreen, S., McBreen, B., Hanlon, L., \& Quilligan, F. 2002, A\&A, 393, L29

McFadyean, A. I., \& Woosley, S. E. 1999, ApJ, 524, 262

Metcalfe, L., Steel, S. J., Barr, P., et al. 1996, A\&A, 315, L105

Mirabel, I. F., Vigroux, L., Charmandaris, V., et al. 1998, A\&A, 333, L1

Moneti, A., Metcalfe, L., \& Schulz, B. 1997, Reference Wavelengths for ISO: CAM and PHOT Filters, ISO Technical Note SAI/97002/DC, http://www.iso.vilspa.esa.es/users/expl_lib/ CAM_list.html

http://www.iso.vilspa.esa.es/users/expl_lib/ISO/ docs/ref_lam/

O'Halloran, B., Metcalfe, L., Delaney, M., et al. 2000, A\&A, 360, 871

O'Halloran, B., Metcalfe, L., McBreen, B., et al. 2002, ApJ, 575, 747

O'Halloran, B., McBreen, B., Metcalfe, L., Delaney, M., \& Coia, D. 2005 [arXiv: astro-ph/0506037]

Okumura, K. 1998, ISOCAM PSF Report http://www.iso.vilspa.esa.es/

Ott, S., Abergel, A., Altieri, B., et al. 1997, In Astronomical Data Analysis Software and Systems VI, ed. G. Hunt, \& H. Payne, ASP Conf. Ser., 125, 34

Ott, S., Gastaud, R., Ali, B., et al. 2001, In Astronomical Data Analysis Software and Systems X, ed. F. R. Jr. Harnden, F. A. Primini, \& H. E. Payne, ASP Conf. Proc., 238, 170

Peeters, E., Spoon, H. W. W., \& Tielens, A. G. G. 2004, ApJ, 613, 986

Pierini, D., Popescu, C. C., Tuffs, R. J., \& Völk, H. J. 2003, A\&A, 409, 907

Popescu, C., Tuffs, R. J., Völk, H. J., Pierini, D., \& Madore, B. F. 2002, ApJ, 567, 221

Richards, P., \& Klaas, U. 2002, in Report on the Scientific Validation of the chopped mode spectroscopy processing in the 
PHT pipeline, v.1. Downloadable from the ISO Data Archive at http://www.iso.vilspa.esa.es/ida/index.html

Roussel, H., Sauvauge, M., Vigroux, L., \& Bosma, A. 2001, A\&A, 372,427

Sage, L. J., Loose, H. H., \& Salzer, J. J. 1993, A\&A, 273, 6

Sanders, D. B., Mazzarella, J. M., Kim, D.-C., \& Surace, J. A. 2003, ApJ, 126, 1607

Sidher, S. D., Griffin, M. J., Davis, G. R., et al. 2000, Icarus, 147, 35

Siebenmorgen, R., Krugel, E., \& Chini, R. 1999, A\&A, 351, 495

Smith, D. A., Herter, T., Haynes, M. P. et al. 1996, ApJS, 104, 217

Soderberg, A. M., Kulkarni, S. R., Berger, E., et al. 2004, Nature, 430, 648

Sofue, Y., Taniguchi, Y., Handa, T., et al. 1990, Publ. Astron. Soc. Jpn, 42, L45

Starck, J.-L., Aussel, H., Elbaz, D., \& others, 1998a, Faint Source Detection with ISOCAM using the PRETI method, in Extragalactic Astronomy in the Infrared, ed. G. A. Mamon, T. X. Thuan, \& J. Tran Thanh Van (Éditions Frontières), Moriond Series in Astrophysics, 509

Starck, J. L., Murtagh, F., \& Bijaoui, A. 1998b. In Image processing and data analysis: The multiscale approach (Cambridge University Press)
Swinyard, B. M., Burgdorf, M. J., Clegg, P. E., et al. 1998, in Infrared Astronomical Instrumentation, ed. A.M. Fowler, Proc. SPIE, 3354, 888

Taniguchi, Y., \& Tamura, S. 1987, A\&A, 181, 265

Taniguchi, Y., \& Noguchi, M. 1991, AJ, 101, 1601

Thuan, T. X., \& Martin, G. E. 1981, ApJ, 247, 823

Thuan, T. X. 1983, ApJ, 268, 667

Vigroux, L., Mirabel, F., Altieri, B., et al. 1996, A\&A, 315, L93

Vigroux, L., Charmandaris, V., Gallais, P., et al. 1999, In The Universe as seen by ISO, ed. P. Cox, \& M. F. Kessler, ESA SP-427, 2, 805

Wainwright, C., Berger, E., \& Penprase, B. E. 2005 [arXiv:astro-ph/0508061]

Weedman, D. W. 1972, ApJ, 171, 5

Weiler, K. W., Panagia, N., \& Montes, M. J. 2001, ApJ, 562, 670

Weiler, K. W., Panagia, N., \& Montes, M. J. 2002, ARA\&A, 40, 387

Wilkinson, P. N., \& de Bruyn, A. G. 1990, MNRAS, 242, 529

Xu, C., Sultentic, J. W., \& Tuffs, R. 1999, ApJ, 512, 178

Yin, Q. F., \& Heeschen, D. S. 1991, Nature, 354, 130

Yin, Q. F. 1994, ApJ, 420, 152

Zezas, A., Fabbiano, G., Rots, A. H., \& Murray, S. S. 2002, ApJ 577, 710 